Cahiers $d u$ MONDE RUSSE

\section{Cahiers du monde russe}

Russie - Empire russe - Union soviétique et États indépendants

$51 / 4 \mid 2010$

Sciences humaines et sociales en Russie à l'Âge d'argent

\title{
Alexandre Skirda, La traite des Slaves
}

\section{Aleksandr Lavrov et André Berelowitch}

\section{(2) OpenEdition}

Journals

Édition électronique

URL : https://journals.openedition.org/monderusse/7358

DOI : 10.4000/monderusse.7358

ISSN : 1777-5388

Éditeur

Éditions de l'EHESS

Édition imprimée

Date de publication : 25 novembre 2010

Pagination : 665-669

ISBN : 978-2-7132-2316-7

ISSN : $1252-6576$

Référence électronique

Aleksandr Lavrov et André Berelowitch, "Alexandre Skirda, La traite des Slaves », Cahiers du monde russe [En ligne], 51/4 | 2010, mis en ligne le 09 décembre 2011, consulté le 02 septembre 2022. URL http://journals.openedition.org/monderusse/7358 ; DOI : https://doi.org/10.4000/monderusse.7358

Ce document a été généré automatiquement le 2 septembre 2022

Tous droits réservés 


\title{
Alexandre Skirda, La traite des Slaves
}

\author{
Aleksandr Lavrov et André Berelowitch
}

\section{RÉFÉRENCE}

Alexandre SKIRDA, La traite des Slaves. L'esclavage des Blancs du VIII ${ }^{\mathrm{e}}$ au XVIII $^{\mathrm{e}}$ siècle. Paris : Max Chaleil, 2010, 230 p.

1 Il est difficile actuellement de vivre en France, de s'intéresser à l'histoire, à plus forte raison d'être historien, et d'ignorer complètement le débat sur l'esclavage. Pour les uns, la traite coloniale, et surtout la traite transatlantique, n'est plus seulement un objet entre autres de la recherche : elle est revendiquée comme repère d'identité et objet de mémoire par les descendants des déportés. Pour les autres, en majorité des historiens, le phénomène de l'esclavage colonial, organisé par les Européens, doit être mis en parallèle avec d'autres phénomènes des Temps modernes. C'est ce que tente de faire le livre de l'historien américain Robert C. Davis, qui porte sur l'esclavage des Européens dans les pays du Maghreb aux XVI $\mathrm{X}^{\mathrm{e}}-\mathrm{XVIII}{ }^{\mathrm{e}}$ siècles. L'auteur, qui n'est pas toujours assez critique à l'égard de ses sources, essaie de montrer qu'il s'agissait d'un groupe de plusieurs dizaines de milliers d'esclaves. Il est intéressant de remarquer que cette dernière étude fut tout de suite traduite en France ${ }^{1}$.

2 Ce n'est sans doute pas un hasard si Alexandre Skirda a tenu à exprimer son approbation pour cet « exemple de [...] démarche d'authentique recherche historique ». Le sous-titre de son livre («L'esclavage des Blancs ») renvoie lui aussi au livre de Davis. Mais alors que celui-ci traite des Temps modernes, la plus grande partie du livre de Skirda est consacrée au Moyen-Âge. Or la notion de "race» n'existait pas dans le monde médiéval d'Occident; mettre l'accent sur la race est donc anachronique. C'est que A.Skirda a évidemment été influencé par l'ouvrage de Bernard Lewis sur la « race » et l'esclavage en pays d'Islam, dont il a fait une lecture quelque peu sélective, pour projeter ensuite les idées de Lewis dans le monde médiéval. Projection illégitime car, pendant le Moyen-Âge, 
les Slaves, capturés et réduits en esclavage par leurs voisins, étaient considérés comme vaincus et perdants (avec toutes les conséquences que cela entraînait), mais pas comme des sous-hommes. C'est là la grande différence avec l'expérience de l'esclavage colonial des Temps modernes, véritable chantier des idéologies racistes.

3 «Les Slaves, dans leurs groupes communautaires, n'avaient pas, à l'origine, de membres privés de la liberté ", écrit Alexandre Skirda. «Il s'était bien créé parmi eux une classe de gens plus opulents, mais tous participaient aux mêmes droits et tous étaient libres » (p.139). L'auteur regarde l'esclavage dans la Rus' médiévale comme imposé par les Vikings, qui pratiquaient le commerce international des esclaves. L'auteur ne mentionne pas l'histoire de l'esclavage russe médiéval (holopstvo), non plus que les débats entre historiens sur les parts respectives des esclaves étrangers et moscovites au sein des différentes catégories de holop. Selon Skirda, les Slaves auraient été victimes d'une chasse à l'homme menée à partir du haut Moyen-Âge, d'abord par les Vikings et les Allemands, puis par les Génois, les Tatars et les Ottomans. Mais ce sont surtout les musulmans et les juifs qui attirent l'attention de l'auteur, à cause de l'énergie particulière dont ils auraient fait preuve dans ce commerce.

Les pages de son livre consacrées aux Tatars de Crimée mériteraient de servir d'exemple dans un séminaire portant sur les stéréotypes et l'usage non critique des sources dans la littérature de vulgarisation contemporaine. Les Tatars sont des "prédateurs permanents ». Ils « ne connaissaient ni l'agriculture, ni les métiers, et ne s'occupaient exclusivement que de l'élevage du bétail et des chevaux». Leur "abominable pédagogie » - l'auteur cite le récit de Sigismond von Herberstein, selon lequel les captifs vieux ou malades étaient livrés aux jeunes Tatars pour être achevés - ne "pourrait produire que des monstres » (p. 172, 177).

5 L'image des marchands juifs est un peu différente. L'auteur reconnaît que la majorité des juifs médiévaux n'avait rien à voir avec le commerce des esclaves. Malgré cela, une corporation spéciale de marchands juifs (les Radânites, comme les appelle l'auteur) est déclarée responsable de l'organisation de la traite. Ici on décèle très clairement l'influence néfaste des idées de Lev Gumilev sur le rôle des juifs au sein de certaines sociétés médiévales. En réalité, les autorités ottomanes n'auraient pas pu céder à des marchands non musulmans le monopole du commerce des esclaves, puisqu'elles auraient dû alors renoncer à convertir ceux-ci à l'islam.

Bien que l'auteur ait connaissance de l'interdit religieux en vertu duquel les juifs ne pouvaient ni vendre des esclaves ou des eunuques, ni procéder à la castration (p. 93), il les accuse d'être des «fabricants d'eunuques" (p. 87). La même accusation (d'organiser la castration des captifs) est portée contre les Arméniens et les moines coptes (p. 125). Selon Skirda, cette opération était souvent effectuée par des médecins spécialisés dans la circoncision. Ces pages du livre sont gênantes, et vérifient parfaitement l'analyse de Sigmund Freud : il associe la peur des juifs à celle de la circoncision, qui à son tour fait penser à la castration (travaux de 1909-1910, Moïse et le monothéisme).

7 La logique interne de l'exposé d'Alexandre Skirda exige que, face aux "méchants", apparaissent les «bons». Ici, ce sont les Cosaques, que l'auteur décrit comme "un mouvement d'autodéfense » (p. 178) slave contre les razzias des marchands d'esclaves; à le lire, les Cosaques seraient très proches des partisans de Mahno. Il s'intéresse surtout aux Cosaques du Dniepr, peut-être parce que les fameuses expéditions navales furent le fait des Cosaques du Don. Il n'est pas étonnant que les prises d'otages et la capture de 
prisonniers par les Cosaques, étudiées récemment par Victor Ostapchuk ${ }^{2}$, ne soient pas mentionnées dans le livre d'Alexandre Skirda.

8 À ce point, plusieurs critiques d'ordre général s'imposent. La première concerne le rôle des religions dans la société médiévale. A. Skirda affirme que le christianisme et l'islam adoptèrent, face à l'esclavage, « un rôle ambigu » (p. 222). Tous les arguments lui sont bons - y compris la citation d'Alexandre Eck, qui indique que l'Église elle aussi possédait des esclaves (p. 161). Mais il faut bien voir la tendance opposée, qui était beaucoup plus forte et plus importante. Il est hors de doute que la christianisation des sociétés médiévales a eu pour effet de réduire le nombre de ceux qu'il était permis de réduire en esclavage. L'islamisation des sociétés conquises par les Arabes ou les Ottomans a joué dans le même sens. Par exemple, dans l'Empire ottoman, un musulman (ou même un non-musulman, sujet de l'Empire) ne pouvait être réduit en esclavage. Du reste, particularité qui semble avoir échappé à l'auteur, les monothéistes (par exemple, les orthodoxes) n'étaient pas les plus menacés, mais bien plutôt ceux qui étaient considérés comme " païens ", par exemple les habitants du Caucase du Nord au début des Temps modernes.

La deuxième critique concerne l'esclavage criméen ou ottoman. L'auteur ignore que l'esclave se voyait très souvent proposer l'affranchissement, à condition de se convertir à l'islam. Ce n'était pas le cas pour tous les esclavages de l'époque moderne : ainsi plusieurs catégories de holopstvo étaient héréditaires. De plus, l'auteur reprend des clichés historiographiques éprouvés, par exemple lorsque traitant de la situation des rameurs aux galères, il la qualifie de "destin des plus cruels» (p.196). Nous avons déjà eu l'occasion d'écrire qu'il serait préférable, avant de s'indigner, de définir les critères. S'il s'agit de l'exploitation des rameurs et de leurs conditions de vie effrayantes, Skirda a raison. Si l'on s'intéresse en revanche aux pressions exercées sur eux pour les contraindre à la conversion (c'est-à-dire à un changement d'identité), la situation des rameurs était moins pénible que celle des maisons nobles, soumises à des pressions plus fortes, et l'on ne peut qu'exprimer son désaccord avec l'auteur.

La lecture attentive de l'ouvrage d'Alexandre Skirda suscite deux réflexions, qui portent d'une part sur le départ des grandes idéologies du champ de la recherche historique (ce qui laisse le champ libre à l'histoire « mémorielle » avec toutes ses dérives), d'autre part sur la communication interne entre les historiens.

11 Même lorsque l'historien qui étudie l'esclavage refuse la terminologie marxiste, cela ne l'autorise pas pour autant à négliger les acquis d'une école historique tout entière, pour laquelle l'esclavage n'est pas un phénomène en soi : il représente soit la base même sur laquelle s'édifie un mode de production (l'esclavagisme), soit une institution au sein d'une société différente, féodale par exemple. On peut rappeler à l'auteur que plusieurs historiens analysent la Rus' pré-mongole comme une société caractérisée par une forte proportion d'esclaves. L'histoire du holopstvo moscovite, alimenté en grande partie par les prisonniers de guerre, est également passée sous silence par l'auteur (ainsi d'ailleurs que son abolition par Pierre le Grand). En revanche, les premières tentatives d'introduction du servage à la fin du $\mathrm{XvI}^{\mathrm{e}}$ siècle sont assimilées par Alexandre Skirda à l'introduction de l'« esclavage » (p. 177), ce qui constitue un grave anachronisme.

On sait que la communication entre les historiens n'est pas toujours facile, surtout lorsqu'ils appartiennent à des branches différentes de la recherche. C'est pourtant cette communication qui leur permet d'éviter les erreurs et les lacunes les plus graves. On dirait que le livre de Skirda a été écrit dans un ermitage spécialement conçu pour éviter 
à l'auteur tout contact avec ses collègues. Il suffisait de consulter un hébraïste pour apprendre que gueniza /guenizah ne signifie pas les "archives", mais l'endroit où l'on déposait provisoirement les fragments de manuscrits religieux en attendant de les enterrer (p. 89). Une courte discussion avec un spécialiste de l'Europe de l'Est lui aurait appris que les Khazars étaient sédentaires, et non pas "nomades» (p.141) et qu'une guerre des Varègues contre les Petchenègues relevait de l'histoire-fiction (p.157). Un slavisant pourrait ajouter que la Chronique des temps passés (dite "de Nestor») ne se confond pas avec les "Chronographes", ou histoires universelles (p. 144), et que Ejsk et Taganrog, fondées respectivement en 1777-1778 et en 1698, ne pouvaient donc pas être des colonies de Gênes (p. 163).

Dans le chapitre des lacunes, il faut citer le livre de l'historien américain Richard Hellie', qui estime que les Moscovites préféraient réduire en esclavage leurs compatriotes, ainsi que la polémique qu'il a déclenchée: les historiens russes ont insisté au contraire sur le pourcentage élevé de prisonniers de guerre parmi les holopy. Alexandre Skirda les ignore, tout comme la seule étude sérieuse consacrée aux eunuques, celle de Maryna Kravets. Lui qui s'intéresse tant aux eunuques aurait pu mettre à profit ses conclusions : M. Kravets montre que les eunuques employés à la cour des khans n'étaient pas des Slaves, mais des Africains, envoyés spécialement à Bahčisaraj par les sultans ottomans ${ }^{4}$.

Sous l'influence de la traite transatlantique, l'auteur privilégie un seul parcours: captivité, réduction en esclavage, exportation vers un pays lointain, avec déracinement culturel et impossibilité de retour. Il oublie cependant qu'il s'agit, sur la frontière ottomane, d'un esclavage temporaire ayant pour but d'obtenir une rançon, auquel cas la question de la conversion ou de l'intégration de l'esclave dans la société ne se posait pas. Pour la même raison, il ne s'intéresse guère à la situation des esclaves en Italie ni aux articles qui la décrivent ${ }^{5}$.

Les fautes d'érudition peuvent sembler mineures en comparaison des erreurs de fond, elles n'en sont pas moins gênantes. Très souvent l'auteur cite les sources d'après les livres d'histoire, et les livres d'histoire d'après les ouvrages de compilation. Lorsqu'on se réfère à Aristote ou à Thucydide, on doit indiquer (comme pour tous les classiques) le chapitre et le paragraphe, et non pas seulement la page d'une traduction (p. 76). Skirda prend le recueil d'articles de Halil Inalcik, Studies in Ottoman social and economic history (Variorum reprints, 1985) pour une revue, et déforme le titre ("social and economical history»: l'histoire à peu de frais?) (p. 173). Les statistiques de captifs que donne Inalcik ne proviennent pas des « archives ottomanes », mais s'appuient en partie sur les estimations de Novosel'skij. Quand Alexandre Skirda cite Inalcik pour confirmer les estimations de Novosel'skij, il présente donc un raisonnement circulaire. Le nom de l'historien polonais Maurycy Horn, translittéré en caractères cyrilliques dans une publication russe, est retranslittéré par l'auteur en caractères latins, ce qui donne « Khorn ».

On ne peut reprocher à Alexandre Skirda de mal connaître le russe ou l'ukrainien, car ses anciens travaux le révèlent traducteur attentif et habile. Comment alors a-t-il pu transformer le hetman Dem'jan Mnohohrišnyj (nom de famille signifiant «qui a beaucoup péché ») en "Monogretchny»? Faut-il comprendre "qui se nourrit uniquement de kacha »? Enfin, lorsqu'il cite la relation de Michalon Litwin, publiée en 1615, il remarque que Litwin visita le Khanat de Crimée en 1640-1643 (p. 176). La relation serait-elle une œuvre d'anticipation?

17 Par-delà ces erreurs de détail, le principal reproche que l'on puisse faire à l'auteur est d'être de parti pris. Certes, les prisonniers tatars et turcs en Moscovie - ou dans l'Empire 
des Habsbourg - étaient beaucoup moins nombreux que les prisonniers moscovites ou impériaux de l'autre côté de la frontière. Mais dans cette guerre frontalière sans fin, les statuts juridiques de tous les prisonniers étaient comparables. Essayer de comprendre la situation dans son ensemble, sans oublier aucune de ses composantes, au moyen d'une analyse transversale se révélera certainement plus fructueux à long terme que de donner libre cours à son indignation. Pour généreuse qu'elle soit, celle-ci ne fait pas bon ménage avec l'histoire.

\section{NOTES}

1. Robert C. Davis, Christian Slaves, Muslim Master - White Slavery in the Mediterranean, the Barbary Coast, and Italy, 1500-1800, Basingstoke: Palgrave MacMillan, 2003 ; Esclaves chrétiens, maîtres musulmans: L'esclavage blanc en Méditerranée (1500-1800), traduit de l'anglais par Manuel Tricoteaux, P. : Babel, 2007.

2. Victor Ostapchuk, "The Human Landscape of the Ottoman Black Sea in the Face of the Cossack Naval Raids ", Oriente Moderno, n.s., XX, (LXXXI), (1), 2001.

3. Richard Hellie, Slavery in Russia, 1450-1725, Chicago-Londres : University of Chicago Press, 1982.

4. Maryna Kravets, "Blacks beyond the Black Sea : Eunuchs in the Crimean Khanate ", in Behnaz A. Mirzai, Ismael Musah Montana and Paul E. Lovejoy, éds., Slavery, Islam and Diaspora, Trenton, NJ : Africa World Press, 2009, p. 21-36.

5. Ines Origo, "The Domestic Enemy. The Eastern Slavs in Tuscany in XIV-XV Centuries", Speculum, 30, 1955, p. 321-366 ; S.M. Stuard, «Urban Domestic Slavery in Medieval Ragusa », Journal of Medieval History, 9, 1983, p. 155-171. 